\title{
Principal problems of femtoscopy of small systems
}

\section{Yu.M. Sinyukov*}

Bogolyubov Institute for Theoretical Physics

E-mail: sinvukovdbitp.kiev.ua

\section{V.M. Shapoval}

Bogolyubov Institute for Theoretical Physics

E-mail: shapovalebitp.kiev.ua

The correlation femtoscopy of small systems with typical sizes about $1 \mathrm{fm}$ or smaller is analyzed. The uncertainty principle plays a crucial role for correlation femtoscopy of such systems. It can be taken into account through the formalism of the partially coherent phases for emitters. The basic principles are illustrated by the simple model of Gaussian-like source. The reduction of the interferometry radii and suppression of Bose-Einstein correlation function for small sources are found.

The Seventh Workshop on Particle Correlations and Femtoscopy

September 20 - 242011

University of Tokyo, Japan

\footnotetext{
${ }^{*}$ Speaker.
} 


\section{Introduction}

The femtoscopic space-time structure of the systems is typically represented in terms of the interferometry radii. They are result of a Gaussian fit of the correlation function defined as a ratio of the two- (identical) particle spectrum to the product of the single-particle ones. In pioneer paper [四] the measured interferometry radii were interpreted as the geometrical sizes of the systems. Later on it was found [ [ 2 , []] that for typical systems formed in experiments with heavy ions the above geometrical interpretation needs to be generalized. The treatment of the interferometry radii as the homogeneity lengths [四, 可] in the systems and crucial suggestion as for femtoscopy scanning of the source radiation in different momentum bins [3] bring the possibility to analyze different parts of the source and explain the behavior of the interferometry radii. In addition, the practical method how to use the final state interactions (FSI) and effects of long-lived resonances to extract the BEcorrelations in relatively large systems created in heavy ion collisions has been proposed [G].

An another challenge, which is still actual, concerns the femtoscopy analysis of relatively small systems created in particle interactions such as $p p$ and $e^{+} e^{-}$where the observed femtoscopic scales are approximately $1 \mathrm{fm}$ or smaller. Here we will analyze the femtoscopy of such small systems accounting for the uncertainty principle which is expressed through partial coherence of the radiation from spatially close emitters.

\section{The basic ideas of the correlation interferometry}

In particle physics the positive correlations between numbers of identical pions with close momenta emitted from an interaction region in proton-antiproton annihilations were found in 1960 by Goldhaber et al (GGLP effect) [ [ ]]. It was understood that the nature of the effect lies in quantum statistics for identical particles demanding the symmetrization of bosonic wave function. Later on Kopylov and Podgoretsky [ $[\mathbf{W}$ found the deep analogy between Hanbury Brown/Twiss (HBT) effect and GGLP one and, in fact, transformed the method of the intensity interferometry telescope to pion interferometry microscope. Below we discuss it in detail.

Let us suppose that two identical bosons (e.g., pions) are emitted from the two space-time points, $x_{1}=\left(t_{1}, \mathbf{x}_{1}\right)$ and $x_{2}=\left(t_{2}, \mathbf{x}_{2}\right)$ and then propagate freely. The wave function of a single particle at the initial time $t_{i}$ is in the configuration representation $\delta^{3}\left(\mathbf{x}-\mathbf{x}_{\mathbf{i}}\right)$. At some time $t$ in the momentum $p=\left(p^{0}=E, \mathbf{p}\right)$-representation it is $\Psi_{x_{i}}(p, t)=\frac{1}{(2 \pi)^{3 / 2}} e^{-i E t} e^{-i \mathbf{x}_{i} \mathbf{p}}$.

In momentum representation $\left(p_{1}, p_{2}\right)$ the two bosons wave function is symmetrized and has the form

$$
\Psi_{x_{1}, x_{2}}\left(p_{1}, p_{2} ; t\right)=\frac{1}{\sqrt{2}(2 \pi)^{3}}\left[e^{i \mathbf{p}_{1} \mathbf{x}_{1}} e^{i \mathbf{p}_{2} \mathbf{x}_{2}}+e^{i \mathbf{p}_{2} \mathbf{x}_{1}} e^{i \mathbf{p}_{1} \mathbf{x}_{2}}\right] e^{-i\left(E_{1}+E_{2}\right) t} .
$$

Then the probability to find the particles with momenta $p_{1}, p_{2}$ is expressed through the scalar product of differences of 4-momenta and 4-coordinates of the two pion emission:

$$
W_{x_{1}, x_{2}}\left(p_{1}, p_{2}\right)=\left|\Psi_{x_{1}, x_{2}}\left(p_{1}, p_{2} ; t\right)\right|^{2} \propto 1+\cos \left[\left(p_{1}-p_{2}\right) \cdot\left(x_{1}-x_{2}\right)\right] .
$$

The attentive reader can notice that the formula (I.2) is derived for the case when radiation of the two particles is not independent: if one boson (with momentum $\mathbf{p}_{1}$ ) comes from the one point (say, 
$\mathbf{x}_{1}$ ), another boson (with momentum $\mathbf{p}_{2}$ ) is emitted from different point $\left(\mathbf{x}_{2}\right)$ and vice versa, but the possibility when the two bosons are emitted from the same point, $\mathbf{x}_{1}$ or $\mathbf{x}_{2}$, is excluded. In the case of independent particle radiation all the possibilities are taken into account. Then one usually demonstrates an idea of the correlation femtoscopy basing on some normalized emission function $\rho(x)$ in the compact region $\mathscr{D}$ as the following (we ignore here possible correlations between coordinates and momenta of the emitted particles):

$$
W_{\mathscr{D}}\left(p_{1}, p_{2}\right)=\int d^{4} x_{1} d^{4} x_{2} \rho\left(x_{1}\right) \rho\left(x_{2}\right) W_{x_{1}, x_{2}}\left(p_{1}, p_{2}\right) \propto 1+\left|\int d^{4} x \rho(x) e^{i q \cdot x}\right|^{2}
$$

where $q=p_{1}-p_{2}$. So, the probability to find the particles with momenta $p_{1}, p_{2}$ at very large times $t \rightarrow \infty$ is expressed through the Fourier image of the emission function. This is the typical basis of the correlation interferometry method allowing one to analyze shape and form of ultra small systems. If $\rho$ is the Gaussian-like emission probability that in some reference frame has the form $\rho(x) \propto \exp \left[-\sum_{i=1}^{3} \frac{x_{i}^{2}}{2 R_{i}^{2}}\right] \delta\left(t-t_{0}\right)$, then $([2.3)$ reads as

$$
W\left(p_{1}, p_{2}\right) \propto 1+\exp \left[-\sum_{i=1}^{3} q_{i}^{2} R_{i}^{2}\right] .
$$

For example, the typical resolving width of the correlation function $\bar{q}=50 \mathrm{MeV}$ corresponds to the size $4 \cdot 10^{-15} \mathrm{~m}=4 \mathrm{Fm}$ (Fermi) $=4 \mathrm{fm}$ (femtometer). The origination of the name of the method - correlation femtoscopy is obvious from these estimates.

Problem however appears when we apply the basic formula ([2.3]) to the system with small number of emitters. For example, if there are the only two different emitting points: $\rho(x)=$ $\frac{1}{2}\left(\delta^{4}\left(x-x_{1}\right)+\delta^{4}\left(x-x_{2}\right)\right)$, then from $\left([2.3)\right.$ follows $\left(\rho_{i}=1 / 2\right)$

$$
W_{x_{1}, x_{2}}\left(p_{1}, p_{2}\right) \propto \sum_{i, j=1,2} \rho_{i} \rho_{j}\left(1+\cos \left[\left(p_{i}-p_{j}\right)\left(x_{i}-x_{j}\right)\right]\right)=1+\cos ^{2}\left[\frac{1}{2}\left(p_{1}-p_{2}\right)\left(x_{1}-x_{2}\right)\right] .
$$

The result is incorrect as we shall show.

To analyze the situation in detail let us consider single particle radiation from two points. If the emission from the point $x_{1}$ gives a quantum state which is distinguishable from one which corresponds to emission from the point $x_{2}$, then it is allowed for one to consider the two amplitudes $A_{i}$ that can be realized with some probabilities $\rho_{i}$ and build the corresponding density matrix:

$$
\rho_{1}: A_{1}(p)=e^{i \mathbf{p \mathbf { x } _ { 1 }}} e^{-i E t} \text { and } \rho_{2}: A_{2}(p)=e^{i \mathbf{p} \mathbf{x}_{2}} e^{-i E t} ; \quad \rho_{1}+\rho_{2}=1 .
$$

Here and below we omit multiplier $(2 \pi)^{-3 / 2}$ since these factors cancel in the correlation function (see below). Note that $W_{x_{1}, x_{2}}(p)=\rho_{1} A_{1}(p) A_{1}^{*}(p)+\rho_{2} A_{2}(p) A_{2}^{*}(p)=1$. In the case of two boson independent radiation from two points there are three different final states (amplitudes) $A_{i j}$ that are realized with corresponding probabilities $\rho_{i j}$ :

$$
\begin{gathered}
\rho_{11}: A_{11}\left(p_{1}, p_{2}\right)=e^{i \mathbf{p}_{1} \mathbf{x}_{1}} e^{i \mathbf{p}_{2} \mathbf{x}_{1}} e^{-i\left(E_{1}+E_{2}\right) t} ; \rho_{22}: A_{22}\left(p_{1}, p_{2}\right)=e^{i \mathbf{p}_{1} \mathbf{x}_{2}} e^{i \mathbf{p}_{2} \mathbf{x}_{2}} e^{-i\left(E_{1}+E_{2}\right) t} ; \\
\rho_{12}: \quad A_{12}\left(p_{1}, p_{2}\right)=\frac{1}{\sqrt{2}}\left(e^{i \mathbf{p}_{1} \mathbf{x}_{1}} e^{i \mathbf{p}_{2} \mathbf{x}_{2}}+e^{i \mathbf{p}_{1} \mathbf{x}_{2}} e^{i \mathbf{p}_{2} \mathbf{x}_{1}}\right) e^{-i\left(E_{1}+E_{2}\right) t} ; \rho_{11}+\rho_{22}+\rho_{12}=1 .
\end{gathered}
$$


If $\rho_{i i}=\rho_{i}^{2}$ and $\rho_{1}=\rho_{2}=1 / 2$ then $\rho_{11}=\rho_{22}=1 / 4$ and $\rho_{12}=1 / 2$. Then the probability to find two particles with momenta $p_{1}, p_{2}$ that emitted independently from the two points $x_{1}$ and $x_{2}$ will be in the case of the Bose-Einstein symmetrization for independent amplitudes

$$
C\left(p_{1}, p_{2}\right)=\frac{W_{x_{1}, x_{2}}\left(p_{1}, p_{2}\right)}{W_{x_{1}, x_{2}}\left(p_{1}\right) W_{x_{1}, x_{2}}\left(p_{2}\right)}=\sum_{i \leqslant j=1,2} \rho_{i j}\left|A_{i j}\right|^{2}=1+\frac{1}{2} \cos \left[\left(p_{1}-p_{2}\right)\left(x_{1}-x_{2}\right)\right] .
$$

It is different from (2.5) because the emission of both quanta from the one point (e.g., $x_{1}$ ) brings no interference while it is interfered according to formula (2.3) which is true only for large number of independent emitters. Therefore the simplest "dedication" of the intensity interferometry method (‥3]) has to be corrected to exclude the double accounting of the contributions of both particles from the same points. This correction is significant if the number of independent emitters is not large.

If the emission from points $x_{1}$ and $x_{2}$ is not independent at all (full coherence), then one cannot use the diagonal matrix density associated with the probabilities $\rho_{1}, \rho_{2}$ but only the pure state: $A_{x_{1}, x_{2}}(p)=A_{1}(p)+A_{2}(p)$. The amplitude of the two identical bosons symmetrized over $p_{1}, p_{2}$ in this case is: $A_{x_{1}, x_{2}}\left(p_{1}, p_{2}\right)=A_{x_{1}, x_{2}}\left(p_{1}\right) A_{x_{1}, x_{2}}\left(p_{2}\right)$ and

$$
C\left(p_{1}, p_{2}\right)=\frac{W_{x_{1}, x_{2}}\left(p_{1}, p_{2}\right)}{W_{x_{1}, x_{2}}\left(p_{1}\right) W_{x_{1}, x_{2}}\left(p_{2}\right)}=1 .
$$

Both these cases of completely independent and fully coherent radiations from points $x_{1}$ and $x_{2}$ can be reproduced in the formalism of partially coherent phases [ $[\mathbb{b}]$. To demonstrate it let us consider the two-point source with coordinates $x_{1}$ and $x_{2}$ and some undetermined phases $\phi\left(x_{i}\right)$ $(i=1,2)$ and express amplitude of single boson emission with momentum $p$

$$
A_{x_{1}, x_{2}}(p)=\left(e^{i \mathbf{p} \mathbf{x}_{1}} e^{i \phi\left(x_{1}\right)}+e^{i \mathbf{p} \mathbf{x}_{2}} e^{i \phi\left(x_{2}\right)}\right) e^{-i E t} .
$$

The symmetrized amplitude of the two-boson radiation is

$$
A_{x_{1}, x_{2}}\left(p_{1}, p_{2}\right)=A_{x_{1}, x_{2}}\left(p_{1}\right) A_{x_{1}, x_{2}}\left(p_{2}\right) .
$$

The probability to register the two identical particles with momenta $p_{1}$ and $p_{2}$ is

$$
\overline{W\left(p_{1}, p_{2}\right)}=<A_{x_{1}, x_{2}}\left(p_{1}\right) A_{x_{1}, x_{2}}\left(p_{2}\right) A_{x_{1}, x_{2}}^{*}\left(p_{1}\right) A_{x_{1}, x_{2}}^{*}\left(p_{2}\right)>
$$

where brackets mean an averaging over events with partially coherent phases (over all events of the emission). Then in the case of random phases, $\left\langle e^{i\left(\phi\left(x_{2}\right)-\phi\left(x_{1}\right)\right)}\right\rangle=\delta^{4}\left(x_{1}-x_{2}\right)$, we get result (2.8) and for fully coherent radiation when $\left\langle e^{i\left(\phi\left(x_{2}\right)-\phi\left(x_{1}\right)\right)}\right\rangle=1$ we get (‥9).

In the case of independent emitters in points $x_{1}, x_{2}$ the above phase average can be presented in the form [ []]:

$$
\left\langle e^{i\left(\phi\left(x_{2}\right)-\phi\left(x_{1}\right)\right)}\right\rangle=\delta^{4}\left(x_{1}-x_{2}\right)=G_{12} \delta\left(t_{1}-t_{2}\right)
$$

where $G_{12}$ is the overlapping integral

$$
G_{i j}=\left|\int d^{3} \mathbf{x} \Psi_{x_{i}}(t, \mathbf{x}) \Psi_{x_{j}}^{*}(t, \mathbf{x})\right| .
$$


As it is demonstrated, an independent emission with fully random phases corresponds to the mixed state with probabilities $\rho_{i}$ for $i$-states. Such a description is possible only if bosons are emitted independently from different points $x_{i}$ in distinguishable/orthogonal quantum states. The latter requirement is satisfied in above case of the flat momentum spectra for each emitter, $f(p)=W(p)=$ const, since the initial quantum states $\delta^{3}\left(\mathbf{x}-\mathbf{x}_{\mathbf{i}}\right)$ taken in different points $i$ are orthogonal.

If the momentum spectrum $f(p)$ is essentially not flat, this corresponds to the wave packet characterized by its center $x_{i}$ and some finite width. One can discriminate between the different states $i$ and $j$ only if they are approximately orthogonal: the overlapping integral (R.14) is small, $G_{i j} \ll 1$. In other words, the distance between the centers of emitters have to be larger than the width of the emitted wave packets. Since the latter is the inverse of the variance $\Delta p$ of the momentum spectra, so $\left(x_{i}-x_{j}\right)^{2} \geq 1 / \Delta p^{2}$. The latter expresses the uncertainty principle: one can discriminate the wave packet $i$ without noticeable violation of the particle spectra, if the measurement that localizes the particle position somewhere inside the sphere with the center $x_{i}$ and the diameter not less then $1 / \Delta p$ is related to the quantum state $i$, but not $j$. So, the distance $\Delta x=\left(x_{i}-x_{j}\right)$ should satisfy to uncertainty principle in the form $\Delta^{2} x \Delta p^{2} \geq 1$. Then the system presented by the superposition of quantum states $A_{i}$ with fully random phases like in Eq. (․ㅣ) , after a phase averaging (2.13) is approximated well by the mixed state with diagonal density matrix $\rho_{i j}$.

In relativistic physics there is another uncertainty principle: the measurement of the particle momenta $p$ has accuracy depending on the duration of the measurement $\delta t: \delta p \sim 1 / \delta t$. So one can measure the time of particle emission without noticeable violation of the momentum spectra with accuracy not better than $1 / \Delta p$ and, so, the $\delta$-function in Eq. ([.13) has to be smeared when one deals with the wave packets. So, for normalized wave packets, one cannot use the random phase approximation if the distance in space and time between emitters is less than the width $1 / \Delta p$ of the wave packet (in units $\hbar=c=1$ ). For example, if $f(\vec{p})=\frac{1}{\left(2 \pi \Delta p^{2}\right)^{3 / 2}} e^{-\frac{\overrightarrow{p^{2}}}{2 \Delta p^{2}}}$, then $G_{12}=e^{-\frac{\Delta p^{2}\left(\vec{x}_{1}-\vec{x}_{2}\right)^{2}}{2}}$ at $t_{1}=t_{2}$ and $\delta\left(t_{1}-t_{2}\right) \Longrightarrow G_{12}^{t} \rightarrow e^{-\frac{\Delta p^{2}\left(t_{1}-t_{2}\right)^{2}}{2}}$. The last term expresses uncertainty principle for momentum-time measurements.

Therefore, the fully chaotic phases, or diagonal density matrix, are possible only under some conditions which we discussed before. For normalized quantum states emitted from the points $i$ and $j$ it can be written as

$$
\left\langle e^{i\left(\phi\left(x_{i}\right)-\phi\left(x_{j}\right)\right)}\right\rangle=G_{i j} e^{-\frac{\Delta p^{2}\left(t_{i}-t_{j}\right)^{2}}{2}}
$$

where $G_{i j}$ is the overlapping integral (2.14), and $\Delta p$ is a variance of the momentum spectrum of emitters.

In opposite case when $\left(x_{i}-x_{j}\right)^{2} \ll 1 / \Delta p^{2}$ the states are indistinguishable and overlapping integral (2.15) $G_{i j} \approx 1$ at $t_{1} \approx t_{2}$ since at $x_{i}=x_{j}$ it is just normalization. Then we come back to the fully coherent emission ([2) which takes place for very closed emitters [Q]. So, in both limited cases of chaotic and fully coherent emission Eq. (2.I5) leads to physically obvious results, and so we will use Eq. (2.15) at any distances between the emitters as accounting for uncertainty principle.

\section{The results in simple model}

Let us consider the simplest non-relativistic case of the simultaneous emission of bosons. Then 
the amplitude of emission of a particle with momentum $\vec{p}$ can be expressed as a superposition of the wave functions $\psi_{x_{i}}(\vec{p})$

$$
A(\vec{p})=c \int d^{3} x_{i} \psi_{x_{i}}(\vec{p}) \tilde{\rho}\left(\vec{x}_{i}\right)
$$

where

$$
\psi_{x_{i}}(\vec{p})=e^{i \vec{p} \vec{x}_{i}} e^{i \varphi\left(\vec{x}_{i}\right)} \tilde{f}(\vec{p})
$$

and momentum spectrum with variance $\Delta p=p_{0}$ is

$$
f(\vec{p})=\tilde{f}^{2}(\vec{p})=\frac{1}{\left(2 \pi p_{0}^{2}\right)^{3 / 2}} e^{-\frac{\vec{p}^{2}}{2 p_{0}^{2}}} .
$$

The distribution function of the centers of emission

$$
\rho\left(\vec{x}_{i}\right)=\left(\frac{1}{2 \pi R^{2}}\right)^{3 / 2} e^{-\frac{\vec{x}_{i}^{2}}{2 R^{2}}}, \quad \tilde{\rho}=\sqrt{\rho} .
$$

Probability of the single particle emission averaged over events with different phase distributions is

$$
\overline{W(\vec{p})}=c^{2} \int d^{3} x_{i} d^{3} x_{j} e^{i \vec{p}\left(\vec{x}_{i}-\vec{x}_{j}\right)} \rho\left(\vec{x}_{i}\right) \rho\left(\vec{x}_{j}\right)\left\langle e^{i\left(\varphi_{i}-\varphi_{j}\right)}\right\rangle f(\vec{p}) .
$$

Phase average (2.55) is

$$
\left\langle e^{i\left(\varphi_{i}-\varphi_{j}\right)}\right\rangle=e^{-\frac{p_{0}^{2}\left(\vec{x}_{1}-\vec{x}_{2}\right)^{2}}{2}} .
$$

and one obtains the following expression for probability of one-particle emission

$$
\overline{W(\vec{p})}=N_{1} e^{-\frac{p^{2}}{2 p_{0}^{2}} \frac{1+8 p_{0}^{2} R^{2}}{1+4 p_{0}^{2} R^{2}}} .
$$

where $N_{1}$ is the normalization constant.

In case of emission of two particles we have probability of emission averaged over events with random phases

$$
\begin{aligned}
& \bar{W}\left(\vec{p}_{1}, \vec{p}_{2}\right)=N_{2} \int d^{3} x_{i} d^{3} x_{j} d^{3} x_{k} d^{3} x_{l} e^{i\left(\vec{p}_{1} \vec{x}_{i}+\vec{p}_{2} \vec{x}_{j}-\vec{p}_{1} \vec{x}_{k}-\vec{p}_{2} \vec{x}_{l}\right)} f\left(\vec{p}_{1}\right) f\left(\vec{p}_{2}\right) \tilde{\rho}\left(\vec{x}_{i}\right) \tilde{\rho}\left(\vec{x}_{j}\right) \tilde{\rho}\left(\vec{x}_{k}\right) \tilde{\rho}\left(\vec{x}_{l}\right) \\
& \cdot\left\langle e^{i\left(\varphi_{1}\left(\vec{x}_{i}\right)+\varphi_{2}\left(\vec{x}_{j}\right)-\varphi_{1}\left(\vec{x}_{k}\right)-\varphi_{2}\left(\vec{x}_{l}\right)\right)}\right\rangle
\end{aligned}
$$

Averaged phase factor is expressed as a sum of three members

$$
\left\langle e^{i\left(\varphi_{1}\left(\vec{x}_{i}\right)+\varphi_{2}\left(\vec{x}_{j}\right)-\varphi_{1}\left(\vec{x}_{k}\right)-\varphi_{2}\left(\vec{x}_{l}\right)\right)}\right\rangle=G_{i k} G_{j l}+G_{i l} G_{j k}-G_{i k} G_{i l} G_{j k}
$$

where the third term removes double account which appears in the sum of the first two terms when $i=j=k=l$.

If one ignores the correction for double account, then

$$
C\left(\vec{p}_{1}, \vec{p}_{2}\right)=\frac{\bar{W}\left(\vec{p}_{1}, \vec{p}_{2}\right)}{\bar{W}\left(\vec{p}_{1}\right) \bar{W}\left(\vec{p}_{2}\right)}=1+e^{-q^{2} R^{2} \frac{4 p_{0}^{2} R^{2}}{1+4 p_{0}^{2} R^{2}}}
$$




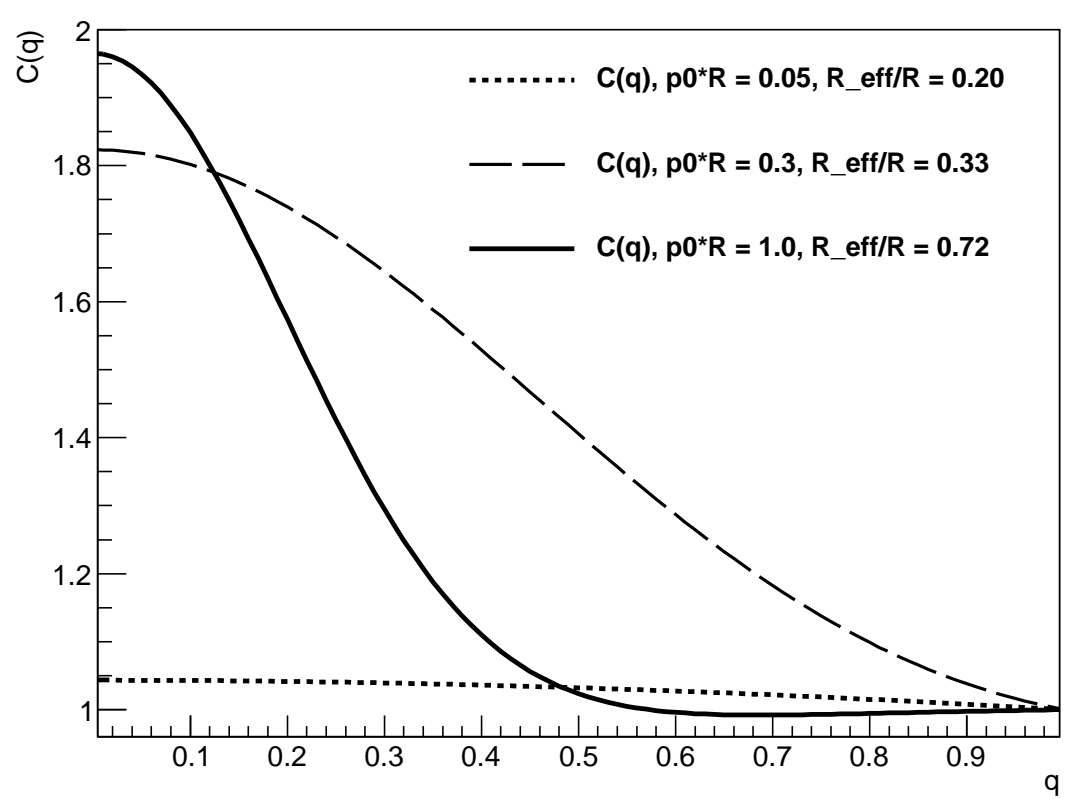

Figure 1: The behavior of the two-particle Bose-Einstein correlation function accounting for the uncertainty principle.

where $q=p_{1}-p_{2}$ and we fix the total normalization in the way which guarantees $C(q) \stackrel{q^{2} \rightarrow \infty}{\rightarrow} 1$. As one can see the observed interferometry radius of the system is reduced as compared to the initial Gaussian radius of the source

$$
\frac{R_{e f f}^{2}}{R^{2}}=\frac{4 p_{0}^{2} R^{2}}{1+4 p_{0}^{2} R^{2}}
$$

The intercept of the correlation function also depends on the size of the system, more correctly on $p_{0}^{2} R^{2}$. To see this one should make correction for double account in Eq. (B.9). The results are presented in Fig. 1. One can see that the intercept of the correlation function tends to unity when the size of the source or momentum spectrum variance tends to zero, $p_{0} R \rightarrow 0$. There are two extreme cases

$$
p_{0} R \gg 1 \quad C\left(\vec{p}_{1}, \vec{p}_{2}\right)=1+e^{-\left(\vec{p}_{1}-\vec{p}_{2}\right)^{2} R^{2}}
$$

which is associated with the standard results, and

$$
p_{0} R \ll 1 \quad C\left(\vec{p}_{1}, \vec{p}_{2}\right) \approx 1
$$

which corresponds to undistinguished positions of the emitters due to uncertainty principle.

\section{Conclusions}

We discussed the principal problems of the interferometry of small sources associated with peculiarities of the correlation femtoscopy formalism in the case of small sources. The new effects appear when one accounts for a partial unobservability of the emitter points due to uncertainty principle. In the case of small sources, $\Delta p^{2} R^{2} \leq 1$, the visible interferometry radii are reduced as compared to the Gaussian radius of the source, and the correlation function is suppressed. The 
detail and full analysis of such effects in the correlation femtoscopy and the consequences for the femtoscopic analysis will be presented in the next publications.

\section{Acknowledgment}

The authors are grateful to S.V. Akkelin for discussions. The research was carried out within the scope of the EUREA: European Ultra Relativistic Energies Agreement (European Research Group: "Heavy ions at ultrarelativistic energies"), and is supported by the National Academy of Sciences of Ukraine (Agreement F3-2012) and by the State fund for fundamental researches of Ukraine (Agreement F33/42-2012).

\section{References}

[1] G.I. Kopylov, M.I. Podgoretsky, Sov. J. Nucl. Phys.: 15 (1972) 219; 18 (1973) 336; 19 (1974) 215.

[2] S. Pratt, Phys. Rev. Lett. 53 (1984) 1219; S. Pratt, Phys. Rev. D 33 (1986)1314.

[3] A.N. Makhlin, Yu.M. Sinyukov, Sov. J. Nucl. Phys. 46 (1987) A. N. Makhlin, Yu. M. Sinyukov, Z. Phys. C 39 (1988) 69345. Yu. M. Sinyukov, Nucl. Phys. A 498 (1989) 151

[4] Yu.M. Sinyukov, Nucl.Phys. A566 (1994) 589c; Yu.M. Sinyukov, in: Hot Hadronic Matter: Theory and Experiment, eds. J. Letessier, H.H. Gutbrod and J. Rafelski (Plenum, New York) 1995, 309.

[5] S.V. Akkelin, Yu.M. Sinyukov, Phys. Lett. B 356 (1995).

[6] Yu.M.Sinyukov, R.Lednicky, J.Pluta, B.Erazmus, S.V.Akkelin Phys. Lett B432 (1998) 248.

[7] Yu.M.Sinyukov, A.Yu.Tolstykh. Z.Phys.C - Particles and Fields 61 (1994) 593.

[8] G.Goldhaber, S.Goldhaber, W. Lee, A. Pais//Phys. Rev. 120 (1960) 325.

[9] R. Lednicky, V.L. Lyuboshits, M.I. Podgoretsky. J.Nucl Phys. 38 (1983) 251. 